OBETS. Revista de Ciencias Sociales Vol. 16, no 1, 2021, pp. 35-50

ISSN: 1989-1385

https://doi.org/10.14198/OBETS2021.16.1.02

\title{
DINÁMICAS DE PARTICIPACIÓN EN LA VEJEZ. DEL ASOCIACIONISMO TRADICIONAL AL ACTIVISMO SIN CARNÉ
}

\author{
PARTICIPATION DYNAMICS IN OLD AGE. FROM TRADITIONAL ASSOCIATIONISM TO \\ NON-MEMBERSHIP ACTIVISM
}

\section{Teresa Amezcua-Aguilar}

Universidad de Jaén, España

mamezcua@ujaen.es

https://orcid.org/0000-0002-8128-8717

\section{Eva Sotomayor-Morales}

Universidad de Jaén, España

esotoma@ujaen.es

https://orcid.org/0000-0002-5579-3859

Cómo citar / Citation: Amezcua-Aguilar, T. y Sotomayor-Morales, E. (2021) "Dinámicas de participación en la vejez. Del asociacionismo tradicional al activismo sin carné". OBETS. Revista de Ciencias Sociales, 16(1): 35-34. https://doi.org/10.14198/OBETS2021.16.1.02

(C) 2021 Teresa Amezcua-Aguilar, Eva Sotomayor-Morales

Este es un artículo de acceso abierto distribuido bajo los términos de la licencia de uso y distribución Creative Commons Reconocimiento 4.0 Internacional (CC BY 4.0) https://creativecommons.org/licenses/by/4.0/deed.es

Recibido: 06/07/20. Aceptado: 16/10/20

\section{Resumen}

Este artículo tiene como objetivo comprender la dinámica de participación social de las personas mayores. El estudio se centra en cómo el contexto y los marcos culturales afectan a las prácticas participativas de las personas mayores. Para ello, realizamos un estudio comparativo entre los casos de Alemania y España como representaciones de dos marcos culturales diferentes. Los hallazgos muestran diferencias en el modelo de participación de los mayores alemanes y españoles. Estas diferencias se sustentan en los roles que cada marco cultural atribuye a las personas mayores y al grado de institucionalización de las estructuras de participación en la vejez.

Palabras clave: Personas mayores; asociacionismo tradicional; nuevas formas de participación social; España; Alemania.

\begin{abstract}
This paper aims to understand the dynamics of social participation of the elderly. The study focuses on how context and cultural frameworks affect the participatory practices of older people. We conducted a comparative study between the cases of Germany and Spain as representations of two different cultural frameworks. The findings show differences in the participation model of the elderly between Germany and Spain. These differences base on the roles that each cultural framework attributes to the elderly and the degree of institutionalization of structures of participation in old age.
\end{abstract}

Keywords: Elderly people; traditional associationism; new ways of social participation; Spain; Germany. 


\section{Extended abstract}

Social participation social creates spaces for interaction between the different spheres of society. Otherwhise, participacion provides communication channels for conflict resolution. It is a tool of social self-regulation wich is symbolically linked to the theoretic and practical rules of democracy. In this paper we conceptualize social participation as any collective action linked to the development of social capital, comprising the whole of activities in public or private institutions and organizations of all kinds, such as associations, political parties and trade unions, among others (Alberich y Espadas, 2014).

This article aims to understand the dynamics of social participation of the elderly in two distinct geopolitical contexts: Spain and Germany. The scientific literature shows that the level and degree of social participation differs by country based on four factors: demographic variables, cultural frameworks, political structures and socioeconomic structures (Fourcade y Schofer, 2016; Putnam, 1995; Schofer y Fourcade-Gourinchas, 2001; Verba y Almond, 1963). This work assumes that contextual variables and cultural frameworks are more relevant in ways of participating than the sociodemographic variable of biological age. The study focuses on describing and explaining how these two variables affect the participatory practices of older people. To this end, we carry out a comparative study between the cases of Germany and Spain as representations of two different cultural frameworks, emphasizing four contextual elements: 1) the influence of aging on the participation of the elderly; (2) the type of participation developed; (3) interests, motivations and preferences; 4) Proposals for the incentive of social participation of the elderly.

\section{Method}

Methodological design is based on a comparative case study in which it is intended to investigate the convergences and divergences of the social participation of the elderly in two geopolitical contexts of study: Spain and Germany. To this aim, a qualitative approach was chosen with the triangulation of three research techniques: the semi-structured (ethnographic) interview, the focus group (hereinafter GF) and the participating observation. The design of the sample covers the most sociologically relevant factors through the inclusion of sample variability strata in the two case studies. The research had a total of 53 participants who responded to two profiles: "expert" and "older people". The experts interviewed are social researchers and social intervention professionals with older people with proven experience in the study material (No. 22). The gender variable was not considered for this sample, with a total of $65 \%$ female experts and $45 \%$ men counting. The sample of elderly people interviewed (No. 11) was selected considering the following criteria: 1) age 60 years and older; (2) be identified as a community leader; 3) social class, considering the level of education (primary, secondary and higher education) and the level of income (reference the Interprofessional Minimum Wage -SMI- of 2016 of each country). The sample size was determined according to the methodological principle of saturation of discourse (Saunders et al., 2018). Two FDs were also performed, one in Germany (No. 9) and one in Spain (No. 8). The training of FGs followed the criteria 1 and 3 described above for interviewees identified as community leaders, replacing criterion 2 with "being a member or participating in an associative entity.

\section{Results and discussion}

Into line with other studies (Shanas et al, 2011; Thomas, 2011), the findings show that age is not a determining variable in the level of participation, as the current generation of older people has the ability to intensify their participation thanks to the availability of free time provided by retirement status. The decline in participation in this age cohort is more associated with the reduction of functional capacity caused by aging processes and associated geriatric syndromes that cause loss of personal autonomy and dependence, with particular mention of mobility limitations. In relation to the time available, the findings show differences between the two study contexts. The time available to the elder Spaniards is contingent on the obligations that the family cultural framework imposes in the provision of care. Older Spaniards prioritize care for the care needs of people dependent on their family environment. The provision of informal care to dependent grandchildren, spouses and large parents takes up much of the time available to older Spaniards. This limitation has a much lower impact on the data collected in Germany. Older Germans assume family care ates, however, they are a major asset in the sustainability of voluntary-dependent community activities that are often aimed at providing care. Regarding to the type of participation, the relevance of both institutional and non-institutional structures aimed at channeling the participation of the elderly is noted. In Spain, the high institutionalization of the social participation of the elderly through the Centers for Active Participation, promotes a type of participation led by the technical teams of local administrations through cultural, leisure and sports activities. This type of participation, aimed at promoting active ageing, satisfies the socialization needs of the elderly, but has a very low impact in terms of the capacity to empower the collective. In Germany on the other hand, the existence of institutional structures aimed at the involvement of older people in community decision-making processes promotes greater civic engagement and political advocacy of their interests as a population group.

Finally, the interest of the elderly is identified in a model of participation more flexible and adapted to their needs. Older people of the 21st century respond to a new profile, with higher levels of health and life expectancy, higher educational and cultural levels, with an interest in facing new activities and learnings and with desire to continue to be socially active. However, older people want to maintain control of this resource and use it according to their personal, family and social priorities and needs. The trend is the search for greater independence and prominence in decisionmaking. For this reason, they seek participation in what we call a "cardless activism". In this type of participation their preferences focus on collective actions that allow them to 
maintain control over the type of activities to be carried out and autonomy for decision-making and responsibilities, highlighting as more valued characteristics: a) temporary flexibility; (b) short-term projects; (c) activities in proximity spaces; and d) involvement in a model of socially productive and transformative activism.

\section{Conclusions}

Findings show differences between the general models of participation of the elderly in Spain and Germany. The social participation of the elder Spaniards has a more hedonistic character, focusing on the satisfaction of personal, psychological, social, cultural needs, etc. On the other hand, the participation of the older Germans includes the citizen

\section{INTRODUCCIÓN}

La participación social construye un espacio de interacción entre las diferentes esferas de la sociedad, vertebrando estas interacciones al proporcionar un canal de comunicación para la resolución de conflictos. Sin embargo, no existe un consenso en su definción teórica debido a la multiplicidad de conceptualizaciones y escalas de medida existentes, lo que dificulta comparar resultados (Levasseur et al., 2010).

En este trabajo se parte de una conceptualización amplia de participación social que incluye toda acción colectiva vinculada con el desarrollo de capital social, comprendiendo el conjunto de actividades en instituciones y organizaciones públicas o privadas de todo tipo, como asociaciones, partidos políticos y sindicatos, entre otros (Alberich y Espadas, 2014). Asimismo, se incorpora la propuesta taxonómica de Levasseur et al. (2010: 2145-2146) basada en dos dimensiones principales: 1) niveles de participación del individuo con otros, y 2) objetivos de estas actividades según su orientación (niveles 1 y 2: satisfacción de necesidades básicas; nivel 3: socializar; nivel 4: consecución de objetivos comunes; nivel 5: ayudar a los demás; y nivel 6: contribuir a la sociedad).

La literatura científica evidencia diferencias significativas en el nivel y grado de participación social según los países (Fourcade y Schofer, 2016; Putnam, 1995; Schofer y Fourcade-Gourinchas, 2001; Verba y Almond, 1963) señalando tres factores relevantes para el desarrollo de este tipo de interacciones: (i) perspective and civic commitment as part of its cultural framework in relation to collective action. Finally, we highlight the preference expressed by the elderly to engage in collective activities in a flexible way, in projects that allow them to continue their lifestyles and personal commitments, respecting their decision-making capacity and autonomy. What we've called a cardless activism. Finally, the promotion of the participation of the elderly must take on different tasks: 1) to promote and support the empowerment of the elderly group; 2) consideration of the elders as protagonistssubject; 3) implementation of participatory methodologies capable of incorporating the social reality of older people; 4) targeted design of recruitment strategies; 5) local planning for comprehensive inclusion; 5) orientation towards comanagement.

las variables demográficas, (ii) los marcos culturales y las estructuras políticas; y (iii) las estructuras socioeconómicas.

En cuanto a las variables sociodemográficas, las que presentan una mayor incidencia en la participación son el nivel educativo, el nivel de ingresos, la edad, el sexo y el estado civil. Los niveles de formación e ingresos medio y medio-alto se relacionan positivamente con el nivel de participación social (Lancee y Van de Werfhorst, 2012). Por otra parte, la disminución de ingresos con la jubilación se asocia a pérdida de oportunidades de participación (Amezcua y Alberich, 2020). Con respecto al sexo, se identifica una menor probabilidad de participación social de hombres que de mujeres (Fuhrer y Stansfeld, 2002), diferencia que se incrementa con el avance de la edad (Desrosiers, Noreau y Rochette, 2004). En cuanto a la edad, los individuos de edad media presentan tasas más elevadas de participación, decreciendo con la edad su intensidad (Cutler y Hendricks, 2000).

Con respecto a los marcos culturales, éstos conforman las bases sobre las que se configuran las identidades colectivas y prácticas sociales normalizadas en cada contexto cultural, actuando como "guiones cognitivos, integrados en las tradiciones institucionales y marcos organizativos que dan forma a los comportamientos y prácticas sociales que se consideran legítimos" (Schofer y Fourcade- Gourinchas, 2001: 808). En este sentido, la participación social implica elecciones por parte de la persona, coherentes y acordes con su identidad (Levasseur, 2010). No obstante, la influencia 
de los marcos culturales en las formas de participación depende de las existencia de estructuras de oportunidad política favorables (Oberschall, 1999, Meyer, 2009), por lo que el nivel y grado de participación ciudadana y el activismo sociopolítico se vincula más con las oportunidades existentes para cambiar la estructura institucional y con el posicionamiento ideológico de las instituciones que detentan el poder, que con los valores e ideologías fruto de los marcos culturales (Schofer y Fourcade-Gourinchas, 2001). A este respecto, el grado de estatismo o de corporativismo de las naciones, como patrones institucionalizados de soberanía política y organización, se asocian a patrones diferenciados de compromiso ciudadano (Fourcade y Schofer, 2016). Las estructuras políticas nacionales europeas pueden clasificarse en cuatro tendencias de menor a mayor intervención estatal, en las que España presenta alto grado de estatismo y bajo corporativismo y Alemania alto grado tanto de estatismo como de corporativismo (Schofer y Fourcade-Gourinchas, 2001: 817).

Finalmente, la estructura socioeconómica ha sido analizada como factor determinante del grado de participación. Diferentes estudios sobre el impacto de la desigualdad social en Europa muestran índices más bajos de participación social para los hogares con menos ingresos en los estados más desiguales (Alarcón, Font y Madrid, 2015; BMAS, 2017; Lancee y Werfhorst, 2012). Francés (2009: 45), profundizando en la influencia de la posición social en las prácticas participativas, señala que la globalización y la generalización de la tecnología informacional y comunicacional en las sociedades posindustriales han incorporado nuevos mecanismos de estratificación más allá de la reproducción de clases y que tienen que ver con cómo los individuos establecen sus relaciones y con la diversidad de roles que deciden adoptar en el marco de su entorno.

\section{OBJETIVOS}

Partiendo de la descripción de las tres categorías de análisis citadas, planteamos la hipótesis de que las variables contextuales y los marcos culturales tienen una mayor influencia en la participación social que la edad biológica. El objetivo general es analizar la forma en que estas dos variables inciden en las prácticas participativas de las personas mayores. A través de un estudio comparativo entre Alemania y
España como representaciones de marcos culturales diferentes, se plantean como objetivos específicos: (1) analizar la influencia del envejecimiento en el tipo de participación; (2) analizar los intereses, motivaciones y preferencias vinculadas a la participación de los adultos mayores; (3) realizar propuestas para la incentivación de la participación de los adultos mayores.

\section{METODOLOGÍA}

Al objeto de indagar en la hipótesis, en este estudio se examinan los discursos sobre la participación social desde una doble perspectiva, la de las personas mayores y la de los expertos en intervención con este grupo. Ambos conjuntos de informantes expresan sus creencias, conceptualizaciones y experiencias como sujetos y objetos directos e indirectos de diferentes vivencias de participación social. Los datos para este estudio se extraen de un trabajo más amplio sobre las dinámicas de la participación de las personas mayores, centrándonos en su dimensión social, cívica y psicológica.

El diseño metodológico se basa en un estudio comparado de casos en el que se examinan las convergencias y divergencias de la participación social de las personas mayores en dos contextos geopolíticos de estudio: España y Alemania. Los contextos concretos para el trabajo de campo se sitúan en las regiones del sur de ambos países, en la comunidad autónoma de Andalucía y el estado federado de Baden-Wurtemberg, respectivamente. Las técnicas de investigación grupal con personas mayores se llevaron a cabo en las ciudades de Essligen (Alemania) y Jaén (España), poblaciones de tamaño medio, urbanas, con alta tasa de envejecimiento y cuyos sectores económicos principales son el agrícola (primario); y el turismo y la administración pública (terciario).

Debido a la necesidad de indagar en la subjetividad del fenómeno, se ha optado por un enfoque cualitativo a través de la triangulación de tres técnicas de análisis: la entrevista semiestructurada (etnográfica), el grupo focal (en adelante GF) y la observación participante. Cada técnica permitió una aproximación diferente al objeto de estudio. A través de la entrevista etnográfica se recabaron datos relativos a aspectos como los significados atribuidos, las motivaciones, las distintas aproximaciones ideológicas, las valoraciones 
personales, las emociones, etc. (Díaz-Bravo, et al., 2013). El GF, como entrevista grupal, permitió el surgimiento de debate y discusión sobre los temas planteados, el desvelamiento de consensos y disensos, así como la generación de nuevas ideas (Rabiee, 2004). La observación participante, como técnica complementaria, proporcionó la recogida de datos sobre las interacciones y procesos que se producen entre los individuos y los grupos y las formas en que sus respectivos marcos culturales los modulan (Kawulich, 2006).

El diseño de la muestra abarca los factores sociológicamente más relevantes a través de la inclusión de estratos de variabilidad muestral en los dos casos de estudio. La investigación contó con un total de 56 participantes que respondieron a dos perfiles: "experto" y "persona mayor". Los expertos entrevistados son investigadores sociales y profesionales de la intervención social con personas mayores con contrastada experiencia en la materia de estudio (Vid. Tablal). La muestra de personas mayores entrevistadas se seleccionó considerando los criterios: 1) edad 60 años y más; 2) ser líder comunitario; 3) clase social, considerándose el nivel educativo (primaria, secundaria y estudios superiores) y el nivel de ingresos (referencia al correspondiente Salario Mínimo Interprofesional-SMI de 2016). El tamaño de la muestra se determinó siguiendo el principio metodológico de saturación del discurso (Saunders et al., 2018). Se realizaron también dos GF, uno en Alemania y otro en España. La formación de los GF siguió los criterios 1 y 3 descritos anteriormente para los entrevistados identificados como líderes comunitarios, sustituyendo el criterio 2 por "ser miembro o participar en una entidad de tipo asociativo". Para esta muestra se buscó la representatividad de ambos sexos (Vid. Tablas 2 y 3 ).

El trabajo de campo se llevó a cabo en dos fases. En la primera se realizaron las entrevistas a informantes clave utilizando un guion semiestructurado desde el que se abordaron preguntas abiertas en bloques temáticos como la conceptualización de la vejez, las condiciones de vida de las personas mayores, la conceptualización de la participación, el compromiso ciudadano de las personas mayores y las oportunidades y barreras para la participación de este grupo poblacional. Esta primera fase permitió la detección de categorías emergentes y enfoques de afrontamento que fueron incorporadas al guion de los GF integrados por personas mayores miembros de asociaciones. Las entrevistas y GF fueron dirigidas por la investigadora principal. Los resultados fueron registrados con grabadora de voz para su posterior transcripción. Partiendo de los discursos se determinaron los significados latentes de los relatos, reestructurando las categorías de análisis y las definiciones de las mismas. Se utilizó el apoyo técnico del software Atlas.ti V8 para la codificación de temas, conceptos y procesos, así como para la sistematización del enraizamiento (E) y densidad (D) de los resultados.

Tablal. Muestra de expertos/as

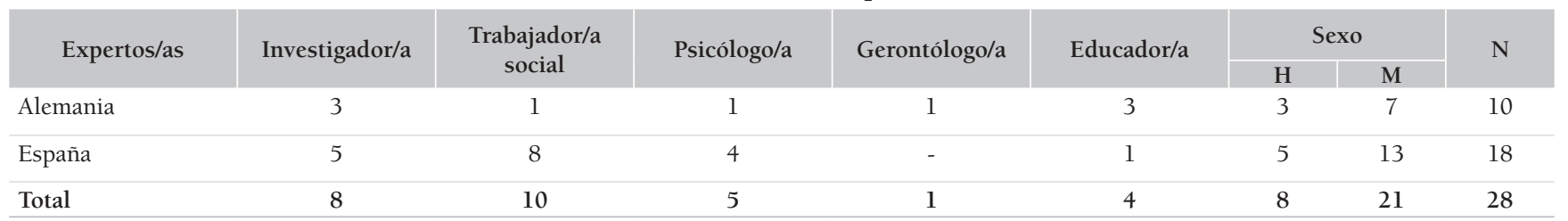

Tabla 2. Muestra de líderes comunitarios mayores

\begin{tabular}{|c|c|c|c|c|c|c|c|c|}
\hline \multirow{2}{*}{ Categoría } & \multirow{2}{*}{ Clase social } & \multirow{2}{*}{ Edad } & \multirow{2}{*}{ Ingresos } & \multicolumn{2}{|c|}{ País } & \multicolumn{2}{|c|}{ Sexo } & \multirow{2}{*}{$\mathrm{N}$} \\
\hline & & & & España & Alemania & $\mathrm{H}$ & M & \\
\hline \multirow{3}{*}{$\begin{array}{l}\text { Líderes } \\
\text { comunitarios }\end{array}$} & Precariado & $\geq 60$ & $\mathrm{I} \stackrel{\mathrm{m}}{=}$ Ingreso $\leq 1 \times \mathrm{SMI}$ & 2 & 1 & 1 & 2 & 3 \\
\hline & Clase media & $\geq 60$ & $\mathrm{I} \stackrel{\mathrm{m}}{=}$ Ingreso $\leq 4 \times \mathrm{SMI}$ & 4 & 2 & 3 & 3 & 6 \\
\hline & Clase alta & $\geq 60$ & $\mathrm{I} \stackrel{\mathrm{m}}{=}$ Ingreso $\geq 4 \times \mathrm{SMI}$ & 1 & 1 & 1 & 1 & 2 \\
\hline Total & & & & 7 & 4 & 5 & 6 & 11 \\
\hline
\end{tabular}


Tabla 3. Muestra de personas mayores

\begin{tabular}{|c|c|c|c|c|c|c|c|c|}
\hline \multirow{2}{*}{ Categoría } & \multirow{2}{*}{ Clase social } & \multirow{2}{*}{ Edad } & \multirow{2}{*}{ Ingresos } & \multicolumn{2}{|c|}{ País } & \multicolumn{2}{|c|}{ Sexo } & \multirow{2}{*}{$\mathrm{N}$} \\
\hline & & & & España & Alemania & $\mathrm{H}$ & M & \\
\hline \multirow{3}{*}{$\begin{array}{l}\text { Líderes } \\
\text { comunitarios }\end{array}$} & Precariado & $\geq 60$ & $\mathrm{I} \stackrel{\mathrm{m}}{=}$ Ingreso $\leq 1 \times \mathrm{SMI}$ & 2 & 1 & 1 & 2 & 3 \\
\hline & Clase media & $\geq 60$ & $\mathrm{I} \stackrel{\mathrm{m}}{=}$ Ingreso $\leq 4 \times \mathrm{SMI}$ & 4 & 2 & 3 & 3 & 6 \\
\hline & Clase alta & $\geq 60$ & $\mathrm{I} \stackrel{\mathrm{m}}{=}$ Ingreso $\geq 4 \times \mathrm{SMI}$ & 1 & 1 & 1 & 1 & 2 \\
\hline Total & & & & 7 & 4 & 5 & 6 & 11 \\
\hline
\end{tabular}

\section{EL TIPO DE PARTICIPACIÓN DE LAS PERSONAS MAYORES. PARTICIPACIÓN DIRIGIDA INSTITUCIONALMENTE EN ESPAÑA Y PARTICIPACIÓN EMPODERADORA EN ALEMANIA}

Existen evidencias científicas de declive en el nivel de participación a partir de la edad media hasta la vejez (Cutler y Hendricks, 2000), sin embargo, los resultados de este estudio respaldan otras teorías que inciden en la pérdida funcional como causa de la disminución en las relaciones sociales (Shanas et al, 2011; Thomas, 2011). Los hallazgos muestran que no se producen descensos en la participación de las personas mayores si éstas mantienen su capacidad funcional, siendo la pérdida de autonomía y la dependencia el factor principal en la disminución de las relaciones sociales y no la edad biológica.

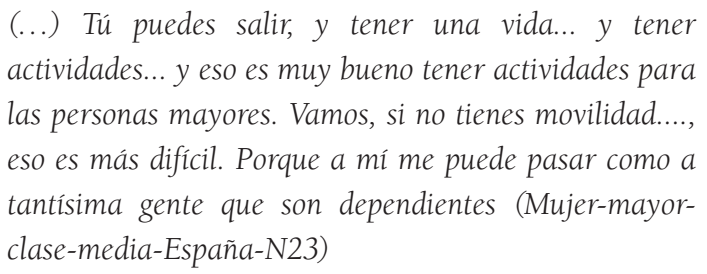

En cuanto a la influencia de los marcos culturales, los datos obtenidos muestran patrones de prácticas participativas diferenciados entre Alemania y España debidas a la atribución de funciones en el ámbito familiar y comunitario y los diferentes modelos de usos del tiempo que éstas implican. Coincidiendo con investigaciones previas (Rodríguez-Cabrero et al., 2013), el tiempo para los mayores españoles está supeditado a las obligaciones que el marco cultural familista impone en la prestación de cuidados. Este grupo prioriza la implicación en la atención de las necesidades de personas dependientes de su entorno familiar. La prestación de cuidados informales a nietos, cónyuges y padres grandes dependientes ocupan buena parte del tiempo disponible de los mayores españoles, limitando el tiempo disponible para la participación social, como ejemplifica esta cita relativa a los costes de la participación:

\section{El mayor coste de participar, el tiempo, porque yo me organizo en función de los nietos (Hombre-mayor- precariado-España-N38)}

Esta limitación presenta una incidencia muy inferior en los datos recabados en Alemania. Los mayores alemanes asumen con menos frecuencia tareas de cuidados en el ámbito familiar, sin embargo, son un activo de gran peso en la sostenibilidad de actividades comunitarias que dependen del voluntariado y que en muchas ocasiones están dirigidas a la prestación de cuidados. En este sentido las personas mayores prefieren participar socialmente con otros mayores, con quienes comparten intereses personales, pero también roles sociales funcionales.

En cuanto al tipo de participación, las personas mayores alemanas, principalmente de las clases medias y medias-altas, normalizan el activismo político y cívico en sus prácticas participativas, afirmando estar comprometidas con el desarrollo de su comunidad con una motivación ético-ideológica. En Alemania los mayores en general tienen un gran peso en la sostenibilidad de actividades de la comunidad que dependen del voluntariado, sobre todo en la atención y prestación de cuidados. En su mayor parte se trata de acciones colectivas generadas por grupos informales con baja institucionalización.

\footnotetext{
Nos juntamos unas cuantas amigas y nos dijimos: estamos retiradas y tenemos tiempo suficiente, ipor qué no hacemos de abuelas de otros niños, en otras familias que no tienen abuelos o algo así? ¡Me gusta la idea! Es adoptar el rol de abuela para personas extrañas. Y se
} 


\begin{abstract}
lo digo a mis amigas y dicen: ¡Ah! ¡Es una gran idea! Estoy sola, mi familia está en Hamburgo, a $650 \mathrm{~km}$ de aquí, y yo aquí sola. Me gustaría tener un nieto aquí para cuidar. Así que montamos un círculo de abuelitas y nos pusimos un nombre: "Las Buenas Abuelas" (risas). Y ahora somos una organización (Mujer-mayor-clasemedia-Alemania-N30)
\end{abstract}

Uno de los ejemplos más destacados en la forma de acción social solidaria de las personas mayores en Alemania es la de las cooperativas ciudadanas de mayores. Surgidas en Baden-Württemberg como una forma de participación socialmente productiva y que favorece sus opciones de empoderamiento. Como explica este informante, se trata de "un modelo de voluntariado que ha sido fundamental para dar un salto cualitativo de un modelo de asistencia segmentada, paternalista y orientada hacia el déficit de los mayores, hacia un modelo de autonomía basado en la autoayuda" (Experto-Hombre-Alemania-N16). Este modelo de participación colectiva desde la autoayuda se basa en la reconstrucción de la imagen social de la vejez con los principios de independencia, autoconfianza, orientación hacia los recursos y práctica de una ciudadanía activa. El objetivo de estas entidades es la dinamización de la comunidad incrementando su potencial, responsabilizándose para ello de la coordinación y planificación de actividades. "La autoayuda y la autorganización se convierten así en un camino y una meta" (Experta-Mujer-Alemania-N21).

En el ámbito de la participación ciudadana, se evidencia la importancia de los "Consejos locales y de distrito de la Tercera edad", órganos de participación ciudadana de las administraciones locales alemanas. Su función principal es asesorar a los órganos políticos, la administración y otras instituciones sobre las soluciones a los diferentes problemas y necesidades de la comunidad. Los miembros de estos consejos son personas mayores voluntarias que trabajan de forma no partidista y aconfesional, lo que les confiere una mayor independencia. Su capacidad de impacto comunitario cobra especial importancia para un colectivo que, tras la jubilación, ha perdido capacidad de incidencia social. Su funcionamiento como órgano de democracia directa favorece el empoderamiento de los mayores, que encuentran en ellos una vía óptima para incluir los intereses del colectivo en las agendas políticas. Los entrevistados alemanes, en este aspecto, destacan la estructura político-organizativa alemana al integrar diversos órganos de participación ciudadana protagonizada por personas mayores. Estas entidades permiten tener incidencia política para los intereses del colectivo en la comunidad.
El Consejo de Mayores de la Ciudad (...) organiza muchas actividades dirigidas a promover la participación, no sólo de los mayores, de toda la comunidad (...) Es un equipo de catorce personas y yo soy presidente desde hace dos años. Cada tema es debatido intensamente. Por ejemplo, sugerir al Ayuntamiento qué áreas para construir nuevas viviendas, para qué podrían ser usadas. Y este es un ejemplo de participación directa (Hombre-mayor-clase media-Alemania-N29)

En cambio, el compromiso político y ciudadano presenta baja relevancia en el discurso de los mayores españoles, circunscribiéndose al voto y la militancia. La participación principalmente es concebida como una actividad motivada por intereses personales y privados y dirigida a la gratificación individual. Para las personas mayores españolas el asociacionismo y el voluntariado se constituyen en vías para continuar integrados socialmente tras la jubilación. Muchas personas mayores se involucran con entidades sociales del tercer sector donde pueden asumir responsabilidades, adoptando un rol de participante-sujeto, generador de capital social.

\footnotetext{
El voluntariado para mí fue una sorpresa, porque primero me dediqué a mis hijos, luego a mi negocio, y nunca se me ocurrió, porque no tenía horas libres. Pero lo que le pasa a mucha gente, que y vi una cola tremenda de emigrantes que están esperando para cenar y pregunté que qué era aquello y me dijeron que era un comedor. Y como lo mío es la cocina y la restauración, entré y me ofrecí (...) y como para mí eso era algo que hago a diario, pues me era más fácil. Y empecé a participar, a participar y... bueno, ahí estoy, y muy satisfecha, porque te digo, que llegas a casa cansada, pero has hecho algo por los demás y eso creo que es importante (Mujermayor-clase-alta-España- N32)
}

Por otra parte, la participación es una oportunidad, sobre todo para las mujeres, para realizar actividades nuevas, nuevos aprendizajes, descubrir capacidades y aptitudes o afrontar retos que no se han podido cumplir durante la adultez. En este sentido, se observa que la existencia de estructuras formales de participación favorece la implicación de las personas mayores en 
los procesos de toma de decisiones comunitarias. La habilitación de este tipo de estructuras promueve la participación de este grupo. Por ejemplo, los mayores españoles tienden más a participar en actividades e iniciativas constituidas previamente, ya que les ofrece un marco de referencia consolidado, como evidencia el siguiente extracto:

Para mí es muy gratificante haber llegado a esta etapa de mi vida, porque yo nunca pensé que iba a hacer lo que estoy haciendo. Participo en la Universidad de Mayores, participo en grupos de voluntariado, participo como escritora en concursos, en la junta directiva, participo en el coro de mayores, en el grupo de teatro del CPA. Así que yo creo que estoy viviendo la etapa más feliz de mi vida, porque yo ya tengo a mis hijos situados, tengo a mi marido ique me dure muchísimos años...! (Mujer-mayorclase-media-España-N34).

No obstante, estas estructuras en el contexto español están principalmente dirigidas a atender las necesidades sociales de las personas mayores desde el paradigma del envejecimiento activo, enfoque ligado a la promoción de la salud. Entidades como los Centros de Participación Activa (en adelante CPA), los antiguamente denominados Hogar del Pensionista, fomentan las interacciones informales y la realización de actividades dirigidas. Estas entidades se constituyen en espacios de socialización, pero su alto grado de institucionalización y el enfoque médico rehabilitador reducen la capacidad de empoderamiento de las prácticas participativas de las personas mayores españolas. En el marco de una participación auspiciada por las administraciones públicas y las entidades sociales, se evidencia la necesidad de implementar mejoras e intensificación de las estructuras de participación para este colectivo que faciliten la autogestión de la demanda, de los recursos y la necesidad de participación social de las personas mayores.

Se constata, en cambio, la visibilidad de órganos de participación como las Juntas directivas de las asociaciones o las Juntas de Gobierno de los CPA. Se trata de instrumentos que funcionan como "entes bisagra" entre la práctica asociativa de los mayores, la administración pública y otras entidades financiadoras. Estos órganos presentan baja incidencia política, pero alta capacidad de influencia en la práctica participativa de las entidades, por lo que son percibidos como instrumentos valiosos para el posicionamiento de intereses en la agenda institucional y de control de la gestión de recursos. No obstante, el diseño y gestión de las programaciones de estos centros recae finalmente en los profesionales de los equipos directivos que gestionan los centros, diseñan los programas y actividades y lideran la participación desde una orientación directiva y paternalista. Este hecho es explicado en el discurso como un interés institucional en dificultar que los individuos participen en la planificación o realización de programas, facilitando en cambio a quienes detentan el poder "educar" o "curar" a los participantes (Arnstein, 1969: 217), lo que implica que el grueso de participantes adopta un papel de usuarios de recursos. Se identifican también déficits de transparencia en los procesos de legitimación democrática de estos órganos, como denuncia la siguiente cita:

Exactamente, jahí iba yo! La Junta anterior, no supo absolutamente nadie que había una Junta. Los amiguetes hicieron la Junta, una señora de X venía una vez al mes y punto. Ahora se fue la señora esa, cumplió; ahora hay otra Junta, exactamente lo mismo: cuatro amiguetes; y no saben ni poner un tablón. (Hombre-mayor-clasemedia-España-N36)

A continuación, se presenta un cuadro-resumen en el que se concretan los principales factores que inciden en la participación de las personas mayores (Vid tabla 4) surgidos en esta investigación. Se estructura en tres niveles -individual, grupal y sistémico- atendiendo a la dimensión en la que tienen capacidad de influencia. 
Tabla 4. Factores de participación de las personas mayores

\begin{tabular}{|c|c|c|c|c|}
\hline Ámbito & Factor & Resultado & España & Alemania \\
\hline \multirow[t]{8}{*}{$\begin{array}{l}\text { Nivel } \\
\text { individual }\end{array}$} & Edad biológica & $\begin{array}{l}\text { La edad biológica no es una variable determinante } \\
\text { en el nivel de participación }\end{array}$ & & $\checkmark$ \\
\hline & Capacidad funcional & Disminución de participación por reducción de la capacidad funcional & & $\checkmark$ \\
\hline & Nivel de ingresos & A mayor nivel de ingresos, más opciones de participación & $\checkmark$ & $\checkmark$ \\
\hline & \multirow[t]{2}{*}{ Motivaciones Sociales } & Participación como vía para seguir integrados socialmente tras la jubilación & & $\checkmark$ \\
\hline & & Oportunidad de nuevos aprendizajes & $\checkmark$ & $\checkmark$ \\
\hline & Motivaciones de salud & $\begin{array}{l}\text { Utilización de recursos comunitarios para la } \\
\text { promoción del envejecimiento activo }\end{array}$ & $\checkmark$ & \\
\hline & Motivaciones ético-cívicas & Existencia de compromiso cívico con la comunidad & & $\checkmark$ \\
\hline & Productividad social & Necesidad de sentirse socialmente productivos & $\checkmark$ & $\checkmark$ \\
\hline \multirow[t]{2}{*}{ Nivel grupal } & \multirow[t]{2}{*}{ Identidad colectiva } & $\begin{array}{l}\text { Sin cohesión grupal porque la variable edad no } \\
\text { es un elemento de identificación }\end{array}$ & $\checkmark$ & $\checkmark$ \\
\hline & & Rechazo a la autoidentificación como "viejo" & $\checkmark$ & $\checkmark$ \\
\hline \multirow{6}{*}{$\begin{array}{c}\text { Nivel } \\
\text { sistémico. } \\
\text { (Marcos } \\
\text { culturales y } \\
\text { sociopolíticos) }\end{array}$} & \multirow[t]{2}{*}{ Atribución de roles sociales } & Prioridad de la prestación de cuidados informales en el entorno familiar & $\checkmark$ & \\
\hline & & Gran peso en la sostenibilidad de actividades comunitarias & & $\checkmark$ \\
\hline & Estructura contextual & El déficit de recursos comunitarios accesibles limitan la participación & $\checkmark$ & $\checkmark$ \\
\hline & Estructuras politicas & Estado de bienestar propicia una participación más empoderadora & & $\checkmark$ \\
\hline & \multirow[t]{2}{*}{$\begin{array}{l}\text { Grado de organización } \\
\text { colectiva }\end{array}$} & $\begin{array}{l}\text { La directividad institucional provoca menor } \\
\text { implicación de las personas mayores }\end{array}$ & $\checkmark$ & \\
\hline & & $\begin{array}{l}\text { La existencia de estructuras de participación ciudadana comunitaria } \\
\text { consolidadas favorece la implicación de las personas mayores }\end{array}$ & $\checkmark$ & $\checkmark$ \\
\hline
\end{tabular}

Fuente: Elaboración propia.

\subsection{Preferencias de las personas mayores en sus prácticas participativas. Activismo sin carné}

El colectivo de personas mayores es considerado como un grupo vulnerable al estimar sus riesgos tanto de salud como de pérdida de relaciones sociales y aislamiento. Los expertos definen la participación de los mayores como poco eficiente en relación con la consecución de beneficios colectivos. Se parte de la premisa de que las estructuras previstas desde las instituciones sociales y públicas para promover la participación de los mayores ya no se ajustan al nuevo perfil de éstos, más formados, más independientes y con nuevas inquietudes, como señala este experto:

Estos nuevos mayores que están llegando son mucho más escépticos y no quieren tener la sensación de ser manipulados, y todas esas cosas que se hacian están ahora mismo un poquito en solfa. (Experto-HombreEspaña-N5).

Se expresa que las vías de participación deben cambiar y adaptarse a la nueva realidad social, a las necesidades, pero también a las preferencias de las personas mayores. Por ejemplo, los expertos reconocen que el diseño de recursos y servicios para las personas mayores se ha centrado principalmente en las situaciones de dependencia, relegando otras necesidades de los mayores, como la de mantener relaciones sociales. Insisten, además, en que "las opciones que hay en este momento de involucrarse en acciones participativas, están la mayoría de ellas orientadas a la clase media" (Experto-Hombre-Alemania-N16). Las actividades que se proyectan requieren muchas veces un nivel educativo mínimo, no ya para llevarlas a la práctica, sino para acceder a la información necesaria. En otras ocasiones implican un gasto económico que no es asumible por todos los mayores. En este sentido se considera que deben encontrarse nuevas estrategias con las que abordar la participación de los mayores, para una eficacia más sostenida de acciones colectivas con diferentes posibilidades de activismo.

Por último, se destaca la importancia de concienciar a la población mayor de los beneficios que les reporta la participación en actuaciones de la comunidad. Ya que, como explica la siguiente cita: "tú sólo participarás 
cuando entiendas que es importante para ti. Así que es necesario tener temas que sean relevantes para la gente" (Experto-Hombre-Alemania-N16). A este respecto se considera que el Trabajo social debe tomar un papel proactivo a la hora de informar a las personas mayores sobre la relevancia de su implicación en los proyectos comunitarios.

\section{Y también intentar explicar a la gente, dejar claro a la gente, que son sus propios asuntos, sus problemas, de los que estamos hablando. (Experto-Hombre- Alemania-N20)}

En cuanto al tipo de participación, los datos recabados nos permiten describir y explicar las preferencias de la actual generación de personas mayores en sus formas de implicarse en acciones colectivas. En líneas generales se muestra predilección por un modelo de compromiso cívico-social flexible, que permita mantener la capacidad de elección del qué, cómo, cuándo, dónde y para qué participar, que hemos denominado "activismo sin carné" (Vid Figura 1).

Los mayores consideran que el activismo y el asociacionismo tradicional requieren un tipo de compromiso que abarca buena parte de la dimensión vital cotidiana. Existe la percepción de que una participación formal "con carné" supone un compromiso demasiado riguroso que los mayores del siglo XXI no están dispuestos a asumir. Los requisitos formales y la exigencia en el nivel de responsabilidades en este modelo de participación son elementos disuasorios para el reclutamiento de los mayores, por lo que muchos no dan el paso de comprometerse en este tipo de iniciativas.

Das la mano y te cogen el brazo. Quieres colaborar un poco, pero te toman todo tu tiempo (...) y cuando tienes miedo de que no te van a dejar hacer las cosas cuando tú quieres, no las empiezas. Esa es la diferencia entre comprometerse y no comprometerse, esa es la respuesta de por qué gente a la que le gustaría comprometerse no lo hace, decide no hacerlo. (Experto-HombreAlemania-N7)

Los adultos mayores del siglo XXI son personas activas, con múltiples intereses, aficiones, responsabilidades y obligaciones a las que atender. Prefieren optar por un activismo en el que el compromiso pueda ser concretado y controlado individualmente. Los nuevos perfiles de personas mayores requieren por tanto nuevas formas de filiación atendiendo a sus usos del tiempo: desarrollo de una vida privada activa sin restricciones temporales en algunos casos; y en otros la necesidad de dedicar tiempo a la prestación de cuidados en el ámbito familiar. La posibilidad de poder elegir el momento en que se participa o no, y cuánto tiempo se dedica a la acción colectiva según unos criterios y necesidades propias, personales y particulares es un elemento trascendental para que las personas mayores decidan comprometerse.

\section{a) Flexibilidad temporal}

Es en este sentido en el que abogan por un tipo de compromiso más flexible, que se compagine mejor con los nuevos perfiles de personas mayores y les permita decidir cuándo y cómo participan. Los mayores prefieren adherirse a iniciativas puntuales, de pequeño recorrido, que no requieran un compromiso a largo plazo. Esto permite tener control sobre el tiempo que se dedica y desvincularse más fácilmente en su caso. Consecuentemente los profesionales perciben que los mayores se involucran más en este tipo de iniciativas, y que además éstas fidelizan mejor el compromiso de los mayores.
Ahora hay más participación en proyectos cortos, a corto plazo, y luego se vuelven a involucrar otra vez. Si tú quieres lo haces, y si no, no. Es completamente tu decisión si vas o no; si vas todas las semanas o cada dos, por lo que no tienes que responsabilizarte ni tener el mismo compromiso que con las asociaciones. Tú vas cuando tú quieres (Experto-Hombre-Alemania-N16)

b) La proximidad como elemento de reclutamiento de las personas mayores

El espacio físico es un elemento fundamental para la elección de la participación. Las limitaciones funcionales que ocasiona el envejecimiento, por una parte, y la multiplicidad de actividades que muchos de ellos mantienen, conllevan la preferencia de participar en el medio más cercano. En consonancia con la literatura científica (Levasseur et al., 2015), los datos permiten establecer una asociación entre una mayor participación social de los adultos mayores con una mayor proximidad de los recursos, tanto públicos como privados. Las personas mayores se vinculan más fácilmente con proyectos e iniciativas en el contexto más próximo físicamente. Donde viven, donde desarrollan su vida, donde están los espacios y lugares de encuentro de su 
vida cotidiana, son las áreas en que los mayores prefieren tomar parte activamente.

\section{Enfrente de mi casa está la asociación de mujeres jaeneras. Pues mira, yo me voy porque me pilla más cerquita que aquí, venir podría venir porque es gratis, pero alli lo tenía enfrente (Mujer-clase-media-España-N27)}

Laproximidad delas estructurasparalaparticipación social es un factor que favorece la implicación de los mayores. El municipio, y más concretamente el barrio se constituyen en los espacios vitales en los que los mayores desean participar. Los expertos concretan que las asociaciones vecinales y las entidades asociativas inscritas en el propio barrio de los mayores son las que concitan un nivel más alto de implicación por parte de éstos. En este caso, barrios activos, con dinámicas comunitarias asociativas, en las que el movimiento vecinal o asociativo es potente, fomentan la participación de los mayores. Por el contrario, un barrio o municipio con un tejido asociativo pobre restringe sus opciones de participación.

\begin{abstract}
Ellos se mueven mucho por lo que pasa a su alrededor; entonces si lo que pasa alrededor les llega, les toca y les satisface, se dejan llevar. Un problema que ocurre tres calles más allá de su barrio, como que lo ven lejos. Ellos viven mucho el día a día de lo que pasa en sus casas, de lo que pasa en su calle, en su barrio. Y si su barrio es un barrio de movimiento, pues ellos se van a sumar, pero si no, no (Experta-Mujer-España-N15)
\end{abstract}

\section{c) Activismo social transformador}

En todo el planeta han surgido en las últimas décadas nuevas formas de participar que difieren bastante del tipo de asociacionismo y voluntariado tradicional. Aunque mayoritariamente estas nuevas maneras de entender la acción social y comunitaria han partido de los jóvenes, han calado también entre las personas mayores, produciéndose un brote de nuevas transferencias comunitarias protagonizadas por éstos. La idea de base de estas iniciativas es la colaboración, el intercambio de recursos personales, aptitudinales o materiales con otros miembros de la comunidad. En este campo, las personas mayores toman un papel de participante-sujeto, reivindicando su valía como parte de la ciudadanía.

En el contexto alemán, por ejemplo, existen numerosas iniciativas de personas mayores que se han con- cretado en entidades asociativas más o menos formales en las que éstos prestan algún tipo de servicio bajo la lógica de la reciprocidad mutua. Estas redes crean tejido social y visibilizan la capacidad del colectivo de seguir contribuyendo al desarrollo comunitario. Muchas de ellas son bancos de tiempo en los que la comunidad se beneficia de las aptitudes y conocimientos de los mayores y éstos se ven rehabilitados como ciudadanos productivos. Algunas son más generalistas y en ellas se intercambian todo tipo de servicios, como los Reparature Café. Otros son más específicos y prestan servicios muy concretos, como las Agencias de Abuelas, en las que personas mayores se ofrecen para cuidar niños adoptando el rol de abuelas/os.

\begin{abstract}
Por ejemplo, el grupo BALANCE, son personas mayores que dijeron "bien, no somos solo viejos, tenemos habilidades, podemos hacer cosas para ti". Por ejemplo, una traducción para ti. Y tú puedes hacer algo por mí, por ejemplo, arreglarme el grifo. Es un intercambio. Es compartir conocimientos (Mujer-mayor-clase-mediaAlemania-N30)

Te hace sentir bien y te llena. Es un beneficio de los dos lados, tanto de lo que se aprende, como de la propia experiencia que se brinda para ayudar o facilitar el desarrollo en la actividad en la que se participa (Hombreclase-media-Alemania-N49)
\end{abstract}

También han surgido nuevas oportunidades de participación social promovidas por las administraciones públicas centradas en la búsqueda de intergeneracionalidad de las prácticas sociales. Las Casas Multigeneracionales, por ejemplo, son espacios diseñados para la realización de actividades conjuntas entre todas las generaciones y orientadas al beneficio mutuo. Su funcionamiento sinérgico favorece la convivencia en la comunidad a través de procesos de toma de decisiones asamblearios donde los participantes deciden las ofertas de servicios a prestar. Los expertos destacan la relevancia de estos centros en el futuro como germen de tejido social, desarrollo comunitario intergeneracional y reducción de desigualdades sociales.

\footnotetext{
Muchos no suelen tener la oportunidad o el apoyo para participar en la vida social de una manera que los haga sentir integrados. La idea de estas casas es que niños, adolescentes, adultos, padres, personas mayores, discapacitados, personas sin discapacidades y personas de diferentes orígenes se reúnan en este lugar y aprendan
} 
unos de otros. Es un lugar de encuentro comunitario $y$ convivencia intergeneracional (Experto-HombreAlemania-N20)

En estas nuevas formas de participación, con una orientación más transformadora, se parte de la premisa de que todos y todas tienen algo que aportar, todos los participantes juegan un rol activo, protagonista de alguna manera. Las personas mayores pueden ejercer como sujetos de la acción y no como meros receptores de recursos y servicios (participante-objeto). Consecuentemente, estas acciones son una forma de transferencia comunitaria con capacidad de empoderar al colectivo de mayores al mismo tiempo que se generan soluciones para problemas comunes.
(...) dicen: no queremos estar oprimidos por nuestro sistema, así que buscamos nuevas formas de vivir juntos, nuevas formas de vida comunitaria. Tratan de inventar formas como los Reparature Caffe, donde no sólo intercambias un café, sino que tú vas y, aunque no tengas mucho dinero, puedes ofrecer, por ejemplo, cinco horas de jardinería y pedir a cambio un par de horas de que le eches un ojo a mis hijos. Puedes intercambiar ideas, habilidades y conocimientos, o puedes ofrecer leer libros a personas mayores y ellas te ayudan cuando tú tienes problemas para reparar una mesa o reparar algo. (Experta-Mujer-Alemania-N8)

Figura 1. Preferencias de participación de las personas mayores.

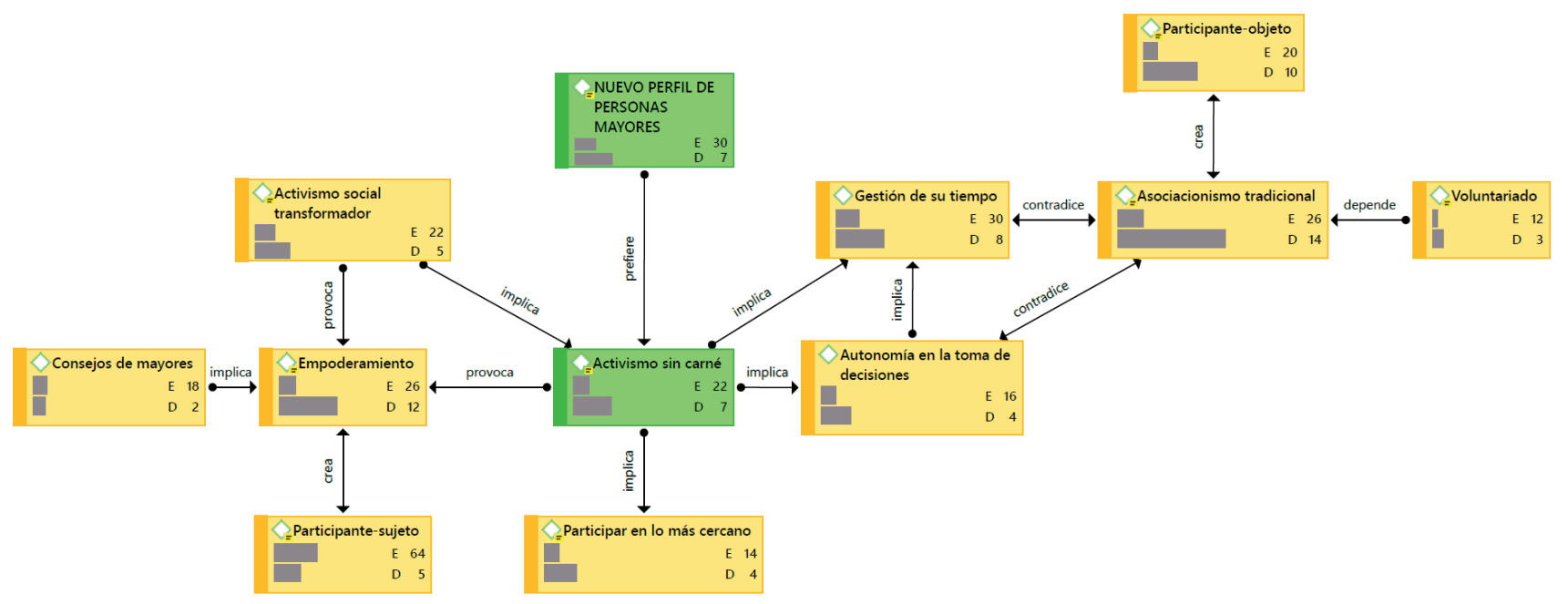

Fuente: Elaboración propia.

\section{DISCUSIÓN}

Coincidiendo con otros estudios (Shanas et al., 2011; Thomas, 2011), los datos recabados muestran que la edad no es una variable determinante en el nivel de participación, ya que la actual generación de personas mayores tiene capacidad de intensificar su participación gracias a la disponibilidad de tiempo libre que les proporciona el estatus de jubilado. Los hallazgos revelan explicaciones a la disminución de la participación social de las personas mayores. En primer término, el descenso de la participación en esta cohorte de edad se asocia más con la reducción de la capacidad funcional que con la edad biológica. Las limitaciones funcionales originadas por los procesos de envejecimiento y los síndromes geriátricos ocasionan pérdida de autonomía personal, especialmente con respecto a la movilidad, reduciendo las oportunidades de participación social.

Coincidiendo con Barnes y Newman (2003) los adultos mayores tienen identidades diversas y distintas experiencias vitales y, por tanto, necesidades, motivaciones y preferencias diversas para participar (Petriwskyj et al., 2014). No obstante, el rechazo a la autoidentificación como persona mayor evidenciados 
en este estudio dificulta una identificación grupal que impulse la acción colectiva, limitándose la capacidad de empoderamiento como grupo social y las oportunidades de participación en actividades colectivas. Aun así, se debe considerar la relevancia de la participación social en la construcción de identidades y representaciones sociales condicionadas por las relaciones de poder materiales y simbólicas en la comunidad (Garrison, 1992; Klandermans et al., 2002; 2004; Oberschall, 1999). En línea con la literatura científica previa (Edwards y Woods, 2017; Jepperson, 2002; Meyer, 2009) los respectivos marcos culturales definen en gran medida los roles desempeñados por la sociedad civil y originan identificaciones grupales y prácticas sociales integradas normativamente. En este sentido, se constata la existencia de círculos de reconocimiento basados en los roles sociales que cada cultura atribuye a las personas mayores. Aunque en ambos contextos el rol atribuido a este grupo implica una contribución social, difieren en el objeto de la misma. En Alemania, esta aportación es un instrumento de sostenibilidad de la comunidad a través del voluntariado, medible en un nivel 6 según la taxonomía de Levasseur et al. (2010). En España, esta contribución se orienta principalmente al apoyo de la familia entendida ésta como institución social básica, lo que sitúa dicho tipo de participación en un nivel mixto entre el nivel 4 (colaborar con otros para alcanzar el mismo objetivo) y el 5 (ayudar a otros).

Por otra parte, la literatura científica señala que el grado y nivel de participación de las personas mayores suele ser resultado de una trayectoria participativa en la adultez que continua e incluso se intensifica en la vejez (McMunn et al., 2009), no obstante, el tiempo libre que proporciona la jubilación puede ser empleado para intensificar o diversificar las actividades en las que se estaba previamente implicado o para iniciar nuevas acciones (Johnson y Bungum, 2008), tanto privadas como públicas, que frecuentemente se concretan en la implicación en acciones colectivas. En otro orden deideas, desde el punto de vista de los beneficios, la acción grupal ofrece una oportunidad de aumentar el capital social de los individuos al utilizar las interacciones sociales como medio para crear este recurso (Bourdieu, 1986). El nivel de asociacionismo es también considerado un indicador de compromiso comunitario y deseo de profundización democrática (Putnam, 1995; Van Deth, 2001; Warren 2001). En este sentido, los hallazgos evidencian una tendencia hacia la promoción de un modelo de activismo social transformador que, en línea con otros autores (Leach, Phillipson, Biggs y Money, 2013; Majón, Ramos y Pérez-Salanova, 2016) identificamos como un compromiso cultural característico de la generación actual de adultos mayores. Este estilo participativo tiende a regirse por criterios de eficacia y cercanía característicos de la evolución de las formas de participación en las sociedades postindustriales (Fouce, 2010), no obstante, los resultados confirman diferencias entre las formas de entender el compromiso cívico en España y Alemania en la misma línea que las señaladas por Francés y Ganuza (2015) al estudiar las actitudes hacia el compromiso político en Europa. El presente trabajo evidencia que el compromiso cívico se manifiesta de forma más evidente y con mayor intensidad en la participación de los mayores alemanes que en la de los españoles. El análisis de los resultados permite asociar esta diferencia a dos factores: el marco sociocultural y el principio de productividad social. El marco sociopolítico del modelo de Estado de bienestar corporativista alemán incita a los mayores a participar en acciones colectivas generadoras de recursos para la comunidad, mientras que el modelo de Estado de bienestar mediterráneo español induce a una participación fortalecedora de la familia como institución social básica. 2) la impronta que las exigencias de productividad social impuestas por el sistema capitalista lleva a la necesidad de sentirse socialmente útiles (Rice, Löckenhoff y Carstensen, 2002), siendo el activismo comunitario el mecanismo para lograrlo.

En cuanto al grado de institucionalización de la participación de las personas mayores, en España ésta se produce mayoritariamente en el marco de entidades de gestión pública, donde se ofrecen una gran cantidad de servicios y prestaciones gratuitas o con precios públicos que son consumidas preferentemente por los mayores de clases bajas y medias. Los mayores en estas entidades participan en los procesos de toma de decisiones desde órganos como los Consejos de Mayores provinciales y de las comunidades autónomas, las Juntas de Gobierno en los CPA, o las Juntas Directivas de las asociaciones. Sin embargo, estos órganos no son percibidos como entidades de relevancia para el empoderamiento de las personas mayores. Las causas parecen radicar en déficits de información por lo que este tipo de participación puede ser descrita, siguiendo las definiciones de Arnstein (1969), como pseudoparticipación o manipulación. 
Por otra parte, a pesar del alto grado de institucionalización de la participación de las personas mayores en España, ésta carece de mecanismos de participación efectivos que faciliten su incidencia en los asuntos de la comunidad. Como señala Martínez-Palacios (2018) el modelo de creciente institucionalización provoca que los profesionales de la intervención social adopten un rol directivo, paternalista-asistencialista que limita la capacidad de empoderamiento del colectivo. En cambio, en el contexto alemán se evidencia que la institucionalización de estructuras de participación consolidadas en las dinámicas democráticas locales proporciona gran capacidad de incidencia sociopolítica en la comunidad. También, el carácter informal de las acciones colectivas favorece una mayor autonomía y genera iniciativas transformadoras.

Finalmente, en los resultados obtenidos en este trabajo destacan la preferencia de las personas mayores por implicarse de manera flexible, con capacidad de decisión y autonomía, en proyectos que les permitan continuar con sus estilos de vida y sus compromisos personales. En este sentido el diseño de políticas dirigidas a fomentar la participación debe tener en cuenta: 1) la consideración de los mayores como protagonistas-sujeto; 2) implementación de metodologías participativas capaces de incorporar la realidad social de las personas mayores; 3) estrategias de reclutamiento focalizadas; 4) planificación local para una inclusión integral; 5) orientación hacia la cogestión.

\section{CONCLUSIONES}

Las personas mayores del siglo XXI responden a un nuevo perfil, con mayores niveles de salud y esperanza de vida, niveles educativos y culturales más altos, interés por afrontar nuevas actividades y aprendizajes y deseos de continuar siendo socialmente productivas. De hecho, son un activo de gran peso en la sostenibilidad de actividades comunitarias que dependen del voluntariado. La jubilación ofrece un recurso fundamental para fomentar su participación: el tiempo. Sin embargo, las personas mayores desean mantener el control de este recurso y utilizarlo de acuerdo a sus prioridades y necesidades personales, familiares y sociales. La tendencia es la búsqueda de mayor independencia y protagonismo en la toma de decisiones. Por ello, buscan participar en lo que denominamos un "activismo sin carné". En este tipo de participación sus preferencias se centran en acciones colectivas que les permitan mantener el control sobre el tipo de actividades a realizar y autonomía para la toma de decisiones y responsabilidades, destacando como características más valoradas: a) la flexibilidad temporal; b) los proyectos a corto plazo; c) actividades en espacios de proximidad; y d) implicación en un modelo de activismo socialmente productivo y transformador.

En definitiva, se resalta la necesidad de fomentar la implicación de las personas mayores en las actividades de la comunidad como un medio de integración social. Para ello la planificación de actuaciones debe diseñarse a partir de un análisis del perfil de los mayores que se pretende involucrar, que exponga sus intereses, necesidades y oportunidades de participación social.

Por otra parte, los hallazgos evidencian diferencias entre los modelos generales de participación de las personas mayores de España y Alemania. La participación social de los mayores españoles se orienta más hacia la satisfacción de necesidades personales, psicológicas y/o sociales, y la realización de actividades para la consecución de objetivos comunes, principalmente en el ámbito familiar. En cambio, la participación de los mayores alemanes incluye el compromiso cívico como parte de su marco cultural en relación a la acción colectiva.

No podemos dejar de señalar como limitación de este trabajo que la metodología empleada y el alcance de la muestra no permiten generalizar resultados ni confirmar la hipótesis de que las variables contextuales y los marcos culturales influyen más en la participación social que la edad biológica. Sin embargo, este estudio supone un avance exploratorio para futuras investigaciones de tipo cuantitativo con una muestra más amplia y representativa.

Quedan muchas incógnitas aún por desvelar para enriquecer el análisis de la participación de las personas mayores. Aspectos como la feminización de la participación en la vejez o la relevancia de las posiciones de clase pueden proporcionar una mejor comprensión del fenómeno. Asimismo, destacamos la necesidad de incorporar en futuras investigaciones la "Marea de Pensionistas y Jubilados", que por criterios metodológicos no fue incluida en este estudio, siendo uno de los movimientos ciudadanos más estables y efectivos y que ha procurado un renovado prestigio social a las personas mayores como agente social activo. 


\section{AGRADECIMIENTOS}

Esta investigación está subvencionada por una Ayuda para contratos predoctorales de Formación del Profesorado Universitario FPU (2014) del Ministerio de Educación, Cultura y Deporte para la realización de la tesis doctoral La desigualdad social y sus correlaciones con la participación de las personas mayores dirigida. Asimismo, para la realización del trabajo de campo, se ha contado con financiación de la Universidad de Jaén: Ayudas para estancias breves del Personal Investigador encaminadas a la obtención del título de Doctor con Mención Internacional (2016).

\section{REFERENCIAS BIBLIOGRÁFICAS}

Alarcón, P., Font, J., y Madrid, E. (2015). Participación Ciudadana y Desigualdad. Diagnóstico y Líneas de Actuación. Madrid: Oxfam Intermond

Alberich, T. y Espadas, A. (2014) Democracia, participación ciudadana y funciones del trabajo social. Trabajo Social Global. Revista de Investigaciones en Intervención Social, 4(6), 3-30.

Amezcua, T., y Alberich, T. Personas mayores: motor de movimientos sociales posindustriales. La marea de pensionistas y jubilados en España y el movimiento Stuttgart 21 en Alemania. Encrucijadas. Revista Crítica de Ciencias Sociales (19). a1902.

Arnstein, S. R. (1969) A Ladder of citizen participation. Journal of the American Institute of planners, 35 (4), 216224. https://doi.org/10.1080/01944366908977225

Barnes, M., Newman, J., Knops, A., y Sullivan, H. (2003). Constituting 'the public'in public participation. Public administration, 81(2), 379-399. https://doi. org/10.1111/1467-9299.00352

BMAS. (2017) Lebenslagen in Deutschland. Der Fünfte Armuts- und Reichtumsbericht der Bundesregierung. Berlin: BMAS.

Bourdieu, P. (1986). The forms of capital. Richardson, J. G. (1986). Handbook of Theory and Research for the Sociology of Education. Connecticut: Greenwood Press, 241-258.

Cutler, S. J., \& Hendricks, J. (2000) Age differences in voluntary association memberships: Fact or artifact. The Journals of Gerontology Series B: Psychological Sciences and Social Sciences, 55(2), 98-107. https://doi.org/10.1093/ geronb/55.2.S98

Desrosiers, J., Noreau, L., \& Rochette, A. (2004). Social participation of older adults in Quebec. Aging clinical and experimental research, 16(5), 406-412. https://doi. org/10.1007/BF03324572

Díaz-Bravo, L., Torruco-García, U., Martínez-Hernández, M., y Varela-Ruiz, M. (2013). La entrevista, recurso flexible y dinámico. Investigación en educación médica, 2(7), 162167. https://doi.org/10.1016/S2007-5057(13)72706-6
Edwards, B., \& Woods, M. (2017) Mobilizing the local: community, participation and governance. Geographies of rural cultures and societies, 173-196). New York/London: Routledge. https://doi.org/10.4324/9781315254487-9

Fourcade, M., \& Schofer, E. (2016) Political Structures and Political Mores: Varieties of Politics in Comparative Perspective. Sociological Science, 3, 413-443. https://doi. org/10.15195/v3.a19

Francés, FJ. (2009). Elementos para el estudio de la estratificación social en las sociedades avanzadas: estrategias operativas. OBETS: Revista de Ciencias Sociales, (3), 43-57. https://doi.org/10.14198/OBETS2009.3.05

Fouce, J. G. (2010). Las viejas y las nuevas formas de participación sociopolítica. Investigación $E$ Desarrollo, 16(2), 262-277. https://doi.org/10.1016/ S0277-9536(01)00111-3

Fuhrer, R., \& Stansfeld, S. A. (2002). How gender affects patterns of social relations and their impact on health: a comparison of one or multiple sources of support from "close persons". Social science \& medicine, 54(5), 811825.

Ganuza, E. y Francés, F. (2015). La participación ciudadana en Europa: un análisis comparado desde los contextos sociopolíticos. OBETS. Revista de Ciencias Sociales, 10(1), 235-260.

Garrison, W. A. (1992). The social psychology of collective action. Frontiers in social movement theory, 1, 53-76.

Jepperson, R. L. (2002) Political modernities: Disentangling two underlying dimensions of institutional differentiation. Sociological Theory, 20(1), 61-85. https:// doi.org/10.1111/1467-9558.00151

Johnson, M. L., \& Bungum, T. (2008). Aging adults learning new avocations: Potential increases in activity among educated baby-boomers. Educational Gerontology, 34(11), 970-996. https://doi.org/10.1080/03601270802042156

Kawulich, B. (2006). La observación participante como método de recolección de datos. Forum Qualitative Sozialforschung / Forum: Qualitative Social Research, 6(2), Art. 43, http://nbnresolving.de/urn:nbn:de:0114fqs0502430

Klandermans, B. (2004). The demand and supply of participation: Social-psychological correlates of participation in social movements. En Snow, D., Soule, S., $\&$ Kriesi, H. The Blackwell companion to social movements, 360-379. https://doi.org/10.1002/9780470999103

Klandermans, B., Sabucedo, J. M., Rodriguez, M., \& De Weerd, M. (2002). Identity processes in collective action participation: Farmers' identity and farmers' protest in the Netherlands and Spain. Political Psychology, 23(2), 235-251. https://doi.org/10.1111/0162-895X.00280

Lancee, B., \& Van de Werfhorst, H. G. (2012) Income inequality and participation: A comparison of 24 European countries. Social science research, 41(5), 11661178. https://doi.org/10.1016/j.ssresearch.2012.04.005

Leach, R., Phillipson, C., Biggs, S., \& Money, A. (2013). Baby boomers, consumption and social change: the bridging generation? International Review of Sociology, 23(1), 104122. http://dx.doi.org/10.1080/03906701.2013.771053 
Levasseur, M., Cohen, A. A., Dubois, M. F., Généreux, M., Richard, L., Therrien, F. H. \& Payette, H. (2015). Environmental factors associated with social participation of older adults living in metropolitan, urban, and rural areas: The NuAge Study. American Journal of Public Health, 105(8), 1718-1725. https://doi. org/10.2105/AJPH.2014.302415

Levasseur, M., Richard, L., Gauvin, L., \& Raymond, É. (2010). Inventory and analysis of definitions of social participation found in the aging literature: Proposed taxonomy of social activities. Social science $\mathcal{E}$ medicine, 71(12), 2141-2149. https://doi.org/10.1016/j. socscimed.2010.09.041

Majón, D., Ramos, P., y Pérez-Salanova, M. (2016). Claves para el análisis de la participación social en los procesos de envejecimiento de la generación baby boom. Psicoperspectivas, 15(2), 53-63. https:// doi.org/10.5027/psicoperspectivas-Vol15-Issue2fulltext-833

Martínez-Palacios,J.2018.Problemasdelainstitucionalización y la profesionalización de la participación en contextos de profundización democrática. Revista Internacional de Sociología 76(1), 089. https://doi.org/10.3989/ ris.2018.76.1.17.95

McMunn, A., Nazroo, J., Wahrendorf, M., Breeze, E., \& Zaninotto, P. (2009). Participation in socially-productive activities, reciprocity and wellbeing in later life: baseline results in England. Ageing and society, 29(05), 765-782. http://dx.doi.org/10.1017/s0144686x08008350

Meyer, J.W. (2009) World society, institutional theories, and the actor. Annual Review of Sociology, 36, 1-20. https://doi. org/10.1146/annurev.soc.012809.102506

Oberschall, A. (1999). Oportunidades y creación de marcos en las revueltas de 1989 en el Este de Europa. En D. M. MCAdam, Movimientos sociales: perspectivas comparadas, 143-181. Madrid: Istmo.

Petriwskyj, A. M., Warburton, J., Everingham, J. A., \& Cuthill, M. (2014). Seniors' motivations for participation in local governance: Evidence from an Australian study. Local Government Studies, 40(2), 240-263. https://doi.org/ 10.1080/03003930.2013.790812

Putnam, R. D. (1995) Bowling alone: America's declining social capital, Journal of Democracy, 6(1), 65-78. https:// doi.org/10.1353/jod.1995.0002

Rabiee, F. (2004) Focus-group interview and data analysis. The Proceedings of the Nutrition Society, 63 4, 655-60. https:// doi.org/10.1079/PNS2004399Rice, C. J.,

Löckenhoff, C. E., \& Carstensen, L. L. (2002). En busca de independencia y productividad: cómo influyen las culturas occidentales en las explicaciones individuales y científicas del envejecimiento. Revista latinoamericana de psicología, 1(1), 133-154.

Rodríguez-Cabrero, G., Rodríguez, P., Castejón, P., y Morán, E. (2013). Las personas mayores que vienen. Autonomía, solidaridad y participación social. Madrid: Fundación Pilares.
Saunders, B., Sim, J., Kingstone, T., Baker, S., Bartlam, B., Burroughs, H. \& Jinket, C. (2018) Saturation in qualitative research: exploring its conceptualization and operationalization. Quality \& quantity, 52(4), 1893-1907. https://doi.org/10.1007/s11135-017-0574-8

Schofer, E., \& Fourcade-Gourinchas, M. (2001). The structural contexts of civic engagement: Voluntary association membership in comparative perspective. American Sociological Review, 66, 806-828. https://doi. org/10.2307/3088874

Shanas, E., Townsend, P., Wedderburn, D., Friis, H. K., Milhoj, P., \& Stehouwer, J. (2017). Old people in three industrial societies. New York, NY: Routledge.

Thomas, P. A. (2011). Gender, social engagement, and limitations in late life. Social science E medicine, 73(9), 14281435. https://doi.org/10.1016/j.socscimed.2011.07.035

Van Deth, J. W. (2001). Soziale und politische Beteiligung: Alternativen, Ergänzungen oderZwillinge? In Politische Partizipation in der Bundesrepublik Deutschland (pp. 195-219). Wiesbaden: VS Verlag für Sozialwissenschaften. https://doi.org/10.1007/978-3-322-99341-0_8

Verba, S., \& Almond, G. (1963). The civic culture: Political attitudes and democracy in five nations. Princeton, NJ: Princeton University Press. https://doi. org/10.1515/9781400874569

Warren, M. R. (2001). Power and conflict in social capital. Beyond Tocqueville: Civil society and the social capital debate in comparative perspective, 169-182.

\section{NOTAS BIOGRÁFICAS}

\section{TERESA AMEZCUA AGUILAR}

Personal Docente e Investigador en el Área de trabajo social y servicios sociales. Dpto. de Psicología. Universidad de Jaén. Trabajadora social, Máster en Dependencia y Autonomía Personal y Máster en Gerontología Social. Dra. en Cuidados Integrales y Servicios Sociales. Sus intereses de investigación giran en torno a los procesos de inclusión y exclusión social, la diversidad y la participación social.

\section{EVA SOTOMAYOR MORALES}

Profesora titular de sociología. Área de trabajo social y servicios sociales. Departamento de Psicología. Universidad de Jaén. Dra. En Ciencias Sociales. IP de la estructura de investigación IN3. Sus intereses de investigación son la inclusión, diversidad y promoción del bienestar 